\title{
The effect of varying numbers of rewarded and nonrewarded trials on subsequent performance in a straight runway'
}

JOHN W. COTTON, UNIVERSITY OF CALIFORNIA, SANTA BARBARA

WINFRED F. HILL, NORTHWESTERN UNIVERSITY, KEITH N. CLAYTON, VANDERBILT UNIVERSITY

Sixty male albino rats were assigned randomly to groups in a design in which one variable was whether rewarded or nonrewarded (latent learning) sessions began the experiment, and the second variable was whether one, two, or four sessions preceded a final rewarded session and extinction session. Extinction speed in a straight runway increased with number of sessions and with initial rewarded rather than nonrewarded sessions. Latent learning sessions facilitated performance on the first rewarded session, the facilitation increasing with number of latent learning sessions.

Previous studies in the straight alley (Jensen \& Cotton, 1961), T-maze (Cotton, Jensen, \& Lewis, 1962; Cotton \& Jensen, 1963), and Skinner box (Cotton \& Jensen, 1961) have demonstrated facilitating effects of a series of extinction trials upon subsequent reacquisition of a learned task. This facilitation may be interpreted as the consequence of habit strength growth occurring as a function of number of trials (Spence, 1956), rather than of number of reinforced trials as Hull (1943) originally postulated.

The process just invoked may also be used to explain latent learning indicated by studies in which trials without reward are followed by later trials in which reward is given and performance on those later trials is superior to that of control groups which did not receive the early unrewarded trials (e.g., Meehl \& MacCorquodale, 1953; Hill, Erlebacher, \& Spear's Experiment 3,1965 ). The present experiment is intended to provide a parametric study of the effect of number of unrewarded trials in training prior to rewarded trials in the straight runway. This study also includes a rewarded comparison group for each latent learning group. Subjects

Sixty male albino rats (Sprague-Dawley strain) approximately 93 to 104 days old were assigned randomly to six groups of 10 each, subject to the condition that one $S$ in each successive six be assigned to each group. Apparafus

The $4 \mathrm{ft}$ long, 4 in. wide runway described by Lewis and Cotton (1960) was employed in this experiment. Response time was measured to the nearest .01 sec for the period from opening of the startboxdoor to crossing of a photocell beam $441 / 2$ in. away.

\section{Procedure}

Let A represent a single day's 30 trial acquisition or rewarded session; $\mathrm{E}$ a corresponding extinction or un rewarded session. Table 2 lists the six groups, forming a two by three factorial design. One variable is latent learning vs conventional learning (A vs $E$ first). The other is the number of sessions prior to a final AE sequence.

Each $S$ was placed on $23 \mathrm{~h}$ food privation for seven days prior to the experiment proper. During the 24th $h$ of this period each $\mathrm{S}$ was tamed for 5 min daily, being handled, allowed to run on an open table top, and allowed to eat $45 \mathrm{mg}$ food pellets of the type (P. J. Noyes lab chow) to be used as rewards later in the experiment. The remainder of the 24th $h$ was used for ad lib feeding in the home cage.

At the end of the seventh day of deprivation, Ss began the experiment proper, receiving 30 trials per day under $22 \mathrm{~h}$ food privation. On each trial $\mathrm{S}$ was placed in the start box and the start doors and goal box opened while $S$ was facing forward. After S left the start box, its door was closed to prevent retracing. When $S$ entered the goal box, its door was closed; $S$ remained there for $\mathbf{3 0}$ sec whether or not food was provided, thus delaying any reward associated with handling. Trials were massed, with $\mathrm{S}$ beginning the next trial immediately upon removal from the goal box following a trial. On A trials there was one food pellet in the food cup. On E trials neither food nor food cup were present in the goal box. If $S$ did not complete a run within $1 \mathrm{~min}$ (particularly as on some early $E$ trials in the latent learning conditions), $E$ carried him through the alley to the goal box and the response time was recorded as $60 \mathrm{sec}$. The following numbers of $S$ in the latent learning groups required this carrying for 15 or more trials on the first training day: Group 1-4, Group 2-2, and Group 3-3. The corresponding numbers for the remaining groups were Groups 4-0, Group 5-1, and Group 6-2.

Results and Discussion

Figure 1 shows mean daily running speeds in each group. An indication of the effect of $\mathrm{E}$ sessions prior to A sessions is obtained by comparing means on the first A day for Group 1 (Day 5), Group 2 (Day 3), and Group 3 (Day 2) to the combined mean for Groups 4, 5 , and 6 (Day 1) in a four group, one way analysis of variance with the last three groups being treated as a single group for statistical purposes. Mean speeds de-

Table 1. Treatment of Groups

(A group number is set equal to each group's design.)

\begin{tabular}{cccc}
\multicolumn{5}{c}{$\begin{array}{c}\text { Number of Sessions Prior to AE Test Sequence } \\
\text { First Session }\end{array}$} & 4 & 2 & 1 \\
\hline E & 1-EEEEAE & $2-E E A E$ & $3-E A E$ \\
$A$ & $4-A A A E A E$ & $5-A E A E$ & $6-A A E$ \\
\hline
\end{tabular}




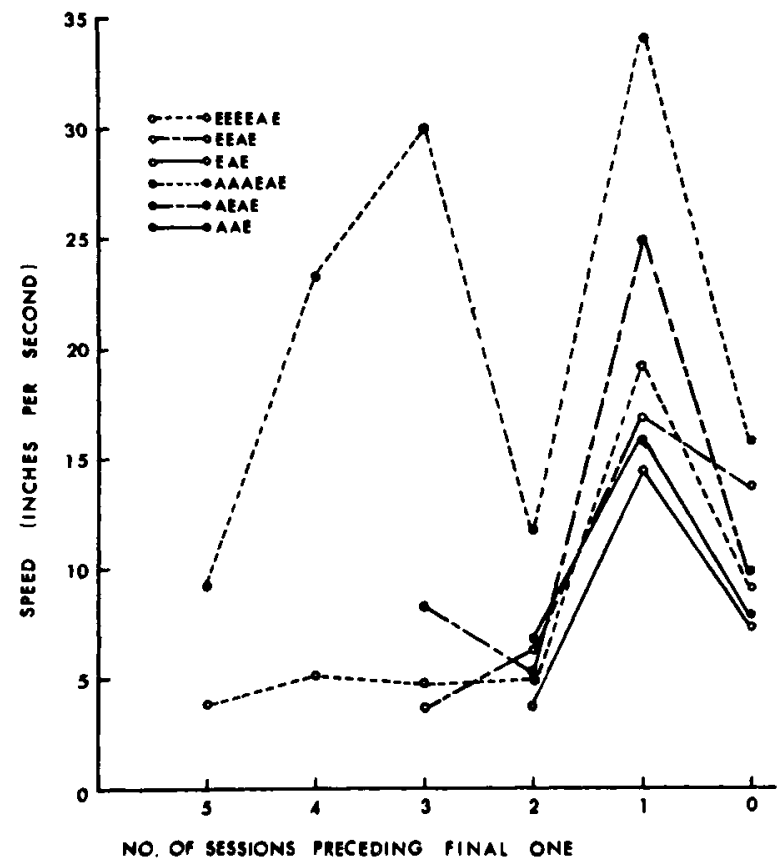

Fig. 1. Mean running speed per session for each group.

cline in order from Group 1 on, indicating that the more $E$ sessions precede the first $A$ the faster will be running speed on $A$; the groups differ significantly $(F=6.27$, $\mathrm{df}=3 / 56, \mathrm{p}<.01$ ).

Use of Fig. 1 for comparison of the groups on the next to the last session shows that they order themselves thus: AAAEAE, AEAE, EEEEAE, EEAE, AAE, and EAE from greatest mean speed on down. Within the set of groups having A first, mean speeds decline with a decrease in number of sessions. The same is true within the set of groups having $\mathrm{E}$ first. The relative superiority of A sessions in facilitating later performance is shown by the fact that, for two groups with equal number of sessions, the one having A first is always superior. This variable dominates over the number of sessions, for EEEEAE is inferior to AEAE, and the analysis of variance reported in Table 2 shows a more highly significant effect for the former variable.

On the final session all groups maintain their immediately previous rank orders except that the EEAE group jumps to the second rank. As a consequence of this jump, an analysis of variance comparable to that of Table 2 yields no significant effect of A vs $E$ first ( $F<1, \mathrm{df}=1 / 54$ ) but significant effects of number of sessions $(F=3.25, \mathrm{df}=2 / 54, \mathrm{p}<.05)$ and of interaction of these two variables $\left(\mathrm{F}=3.21,{ }^{\circ} \mathrm{df}=2 / 54, \mathrm{p}<.05\right)$.

The factlitatory effect of the latent learning sessions may be alternatively attributed to handling, running
Table 2. ${ }^{1}$ Analysis of Variance of Mean Speeds on the Final Acquisition Session

(Next to the Last Day of the Experiment)

\begin{tabular}{lccc} 
Source & $d f$ & $M S$ & $F$ \\
\hline A vs. E first & 1 & 435.03 & $11.45^{* * *}$ \\
No. of sessions & 2 & 299.16 & $7.87^{* *}$ \\
Interaction & 2 & 105.90 & 2.79 \\
Error & 54 & 37.99 & -- \\
\hline
\end{tabular}

${ }^{* *} p<.01 ;{ }^{* * *} p<.005$

${ }^{1}$ The basic datum for this analysis is the total for each individual $s$ of his reciprocal running time's for 30 trials, not converted to in. per sec

through the runway or being carried through it, seeing the endbox and/or any other of the events common to rewarded and nonrewarded trials in the runway. The data suggest the possibility that habit strength grew more slowly under unrewarded trials than rewarded ones. An alternative possibility is that $r_{G}$ for a given reward magnitude (in this case $45 \mathrm{mg}$ ) can be reinstated more rapidly after intervening experience with another magnitude (in this case zero) than it can be established in the first place, an interpretation presented by Spear (1967).

\section{References}

COTTON, J. W., JENSEN, G. D., \& LEWIS, D. J. Spontaneous recovery interval as a factor in reacquisition of T-maze behavior. $J$. exp. Psychol., 1962, 63, 555-562.

COTTON, J. W., \& JENSEN, G. D. Successive acquisitions and extinctions in a T-maze. J. exp. Psychol., 1963, 65, 546-551.

COTTON, J. W., \& JENSEN, G. D. Extinction and reacquisition after differential numbers of acquisition sessions. J. gen. Psychol., 1961, $65,189.195$.

HILL, W. F., ERLEBACHER, A., \& SPEAR, N. E. Reminiscence and forgetting in a runway. J. exp. Psychol., 1965, 70, 201-209.

HULL, C. L. Principles of behavior. New York: Appleton-CenturyCrofts, 1943.

JENSEN, G. D., \& COTTON, J. W. Successive acquisitions and extinctions as related to percentage of reinforcement. J. exp. Psychol., $1960,60,41-49$.

LEWIS, D. J., \& COTTON, J. W. Effect of runway size and drive strength on acquisition and extinction. J. exp. Psychol., 1960, 59, $402-408$.

MEEHL, P. E., \& MacCORQUODALE, K. Drive conditioning as a factor in latent learning. J. exp. Psychol., 1953, 45, 20-24.

SPEAR, N. E. Retention of reinforcer magnitude. Psychol. Rev., 1967, $74,216-234$

SPENCE, K. W. Behavior theory and conditioning. New Haven: Yale University Press, 1965.

Note

1. This experiment was performed at Northwestern University with the support of National Science Foundation NSF G-8706. Preparation of the manuscript was supported by a faculty research grant from the University of California, Santa Barbara. Appreciation is expressed to the Department of Psychology, Indiana University, for hospitality to the senior author during the writing of this article. We also thank John Nielsen and Norman Spear for running $S$ s and analyzing data and Rodney McGinnis for comments during its writing. 